\title{
When a checklist is not enough: How to improve them and what else is needed
}

\author{
Jaishankar Raman, MBBS, MMed, PhD, FRACS, ${ }^{a}$ Nancy Leveson, PhD, ${ }^{\mathrm{b}}$ Aubrey Lynn Samost, SM, ${ }^{\mathrm{c}}$ \\ Nikola Dobrilovic, MD, ${ }^{\mathrm{d}}$ Maggie Oldham, RN, ${ }^{\mathrm{e}}$ Sidney Dekker, PhD, ${ }^{\mathrm{f}}$ and Stan Finkelstein, MD, MS ${ }^{\mathrm{g}, \mathrm{c}}$
}

\section{ABSTRACT}

Objectives: Checklists are being introduced to enhance patient safety, but the results have been mixed. The goal of this research is to understand why time-outs and checklists are sometimes not effective in preventing surgical adverse events and to identify additional measures needed to reduce these events.

Methods: A total of 380 consecutive patients underwent complex cardiac surgery over a 24-month period between November 2011 and November 2013 at an academic medical center, out of a total of 529 cardiac cases. Elective isolated aortic valve replacements, mitral valve repairs, and coronary artery bypass graft surgical procedures $(\mathrm{N}=149)$ were excluded. A time-out was conducted in a standard fashion in all patients in accordance with the World Health Organization surgical checklist protocol. Adverse events were classified as anything that resulted in an operative delay, nonavailability of equipment, failure of drug administration, or unexpected adverse clinical outcome. These events and their details were collected every week and analyzed using a systemic causal analysis technique using a technique called CAST (causal analysis based on systems theory). This analytic technique evaluated the sociotechnical system to identify the set of causal factors involved in the adverse events and the causal factors explored to identify reasons. Recommendations were made for the improvement of checklists and the use of system design changes that could prevent such events in the future.

Results: Thirty events were identified. The causal analysis of these 30 adverse events was carried out and actionable events classified. There were important limitations in the use of standard checklists as a stand-alone patient safety measure in the operating room setting, because of multiple factors. Major categories included miscommunication between staff, medication errors, missing instrumentation, missing implants, and improper handling of equipment or instruments. An average of 3.9 recommendations were generated for each adverse event scenario.

Conclusions: Time-outs and checklists can prevent some types of adverse events, but they need to be carefully designed. Additional interventions aimed at improving safety controls in the system design are needed to augment the use of checklists. Customization of checklists for specialized surgical procedures may reduce adverse events. (J Thorac Cardiovasc Surg 2016;152:585-92)

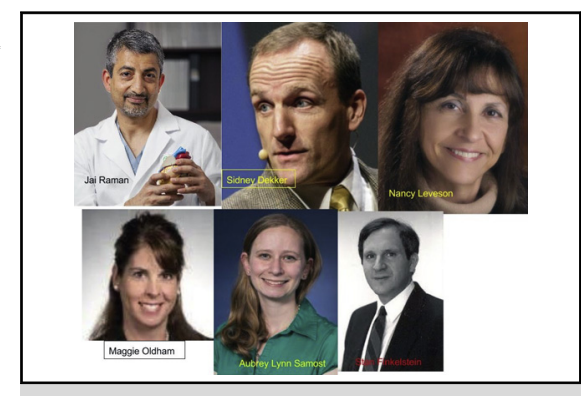

A case for customizing checklists for cardiac surgery.

\section{Central Message}

Routine use of boilerplate checklists may not be enough to minimize perioperative complications in cardiac surgery.

\section{Perspective}

We analyzed 30 adverse events in 380 consecutive cardiac surgery procedures. Despite $100 \%$ compliance with a preoperative surgical checklist, adverse events occurred that were specific to the nuances of cardiac surgery and the associated complexities. We suggest that checklists be customized and modified if they are to be effective in cardiac surgery.

See Editorial Commentary page 593

\footnotetext{
From ${ }^{a}$ Division of Cardiothoracic Surgery, Oregon Health \& Science University, Portland, Ore; ${ }^{b}$ Massachusetts Institute of Technology, Cambridge, Mass; ${ }^{c}$ Engineering Systems, Massachusetts Institute of Technology, Cambridge, Mass; ${ }^{\mathrm{d} C}$ Cardiac Surgery, Rush University Medical Center, Chicago, Ill; ${ }^{\mathrm{e}}$ Healthcare Informatics Consultant, Independent Contractor, Nashville, Tenn; ${ }^{\mathrm{f}}$ Safety Science Innovation Laboratory, School of Social Sciences, Griffith University, Queensland, Australia; and ${ }^{\mathrm{g}}$ Harvard Medical School and Beth Israel Deaconess Medical Center, Boston, Mass.
}

Received for publication Aug 29, 2015; revisions received Dec 9, 2015; accepted for publication Jan 13, 2016; available ahead of print May 7, 2016.

Address for reprints: Jaishankar Raman, MBBS, MMed, PhD, FRACS, Division of Cardiothoracic Surgery, Oregon Health \& Science University, Portland, OR 97239 (E-mail: jairaman24@gmail.com).

$0022-5223 / \$ 36.00$

Copyright (C) 2016 by The American Association for Thoracic Surgery

http://dx.doi.org/10.1016/j.jtcvs.2016.01.022 


\section{Abbreviations and Acronyms \\ CAST $=$ causal analysis based on systems theory \\ $\mathrm{CABG}=$ coronary artery bypass graft \\ EHR = electronic health record \\ $\mathrm{OR}=$ operating room \\ $\mathrm{VAD}=$ ventricular assist device \\ WHO $=$ World Health Organization}

\section{Scanning this QR code will take you to the article title page.}

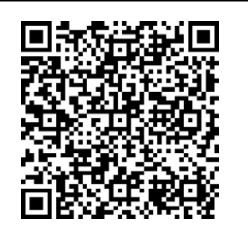

Checklists have been promoted as a way to improve health care outcomes and safety. Studies of the results have been mixed. Some researchers have found impressive decreases in mortality by instituting simple checklists before surgical procedures. For example, Haynes et al. ${ }^{1}$ showed an impressive $35 \%$ decrease in mortality by instituting a simple checklist before every surgical procedure. The SURPASS (Surgical Patient Safety System) checklist also showed greatly improved surgical outcomes. ${ }^{2}$

However, as checklist usage has become more widespread, results have not always been so impressive and have been mixed or inconclusive. ${ }^{3}$ Recently mandated implementation of the World Health Organization (WHO) Safe Surgery Checklist ${ }^{1}$ at all hospitals in Ontario, Canada, failed to show a decline in mortality and morbidity. ${ }^{4}$ Studies began to show that even when $100 \%$ compliance with the checklist was documented, in reality, checklists were completed less than $10 \%$ of the time when operating room (OR) staff were observed. ${ }^{5}$ A recent report by Urbach and colleagues ${ }^{4}$ reported results of the implementation of the WHO surgical checklist in the entire province of Ontario, Canada. We evaluated the effects and use of a standard WHO surgical checklist mandated by the institution, in the setting of complex cardiac surgery. Causal effects of adverse events were also studied, using systems theory.

\section{METHODS}

Data were collected on 380 consecutive complex cardiac surgery cases over a 24-month period, between November 1, 2011 and November 1, 2013 at Rush University Medical Center, a large academic medical center in inner-city Chicago. During this period, 529 cardiac surgery cases were performed and 380 complex cases accounted for $71.8 \%$ of the total caseload. For purposes of uniformity, elective coronary artery bypass graft $(\mathrm{CABG})$ procedures $(\mathrm{n}=77)$, elective simple mitral valve repairs $(\mathrm{n}=22)$, and uncomplicated aortic valve replacements $(\mathrm{n}=55)$ were excluded. Checklist compliance, effectiveness, utilization, and outcomes were evaluated in patients undergoing complex heart surgery. These included emergencies, urgent $\mathrm{CABG}$, multiple valve procedures, combined valve and CABG procedures, aortic surgery, left ventricle reconstruction, ventricular assist device (VAD) implants, and heart transplants. During this period, heart transplantation and VAD implants were restarted as part of heart failure surgery in this institution. All patient and procedure details were made available through the Society of Thoracic Surgeons database maintained by our database manager. This was a retrospective review of cases, approved by the Rush Institutional Review Board.

The cases were observed for completion of the preoperative time-out and the presence of any adverse events. The preoperative checklist used was a modification of the WHO Surgical Safety Checklist and covered the items seen in Table 1. This Surgical Safety Checklist was mandated by the institution and had no specific customization for various specialties. We chose to study outcomes related to the use of the checklists, as a mechanism of process and quality improvement.

Adverse events were defined as anything that resulted in an operative delay, nonavailability of equipment, failure of drug administration, or unexpected adverse clinical outcome. Incidents were identified via direct observation, weekly case reviews, or chart analysis after an Unexpected Occurrence Report was filed by the care team. In addition, weekly meetings were held by the surgical team to uncover adverse events, in an effort to track them and streamline perioperative processes. In terms of the heart transplant procedures, additional investigations were made into processes used for immunosuppression management, organ retrieval, and perioperative protocols. Lines of communication between different intensive care units and clinical services were also assessed.

Adverse events were then analyzed using a system engineering technique called causal analysis based on systems theory (CAST). ${ }^{6}$ CAST, grounded in systems theory, is a more powerful and inclusive analysis technique than the typical root cause analysis used to investigate adverse events. CAST goes beyond individual error and examines the contextual, social, and organizational influences on human behavior. The philosophy behind CAST is that human behavior is influenced by the environment in which it occurs. Assigning blame to doctors, nurses, and technicians does not prevent future incidents unless the environmental determinants of the behavior involved (the systemic factors) are identified and corrected.

CAST (and systems theory in general) is based on the system-theoretic principle that accidents are not only the result of individual system component failures or errors but more generally result from inadequate enforcement of constraints on the behavior of the system components. Examples of safety constraints are that preemptive immunosuppression must be administered to patients before receiving a heart transplant or that all required equipment must be available during cardiac surgery.

The safety constraints are enforced by controls. Controls include such things as physical and logical design to reduce or eliminate common errors, checklists, performance audits, altering the order of steps in a procedure to reduce the risk of skipping some, and changing incentive structures (ie, aligning individual incentives with system-level goals). In general, controls may be physical, procedural, or social. Losses result when the controls are inadequate and flaws in the overall system design and in the interactions among the system components violate the safety constraints. Safety is treated not as a human reliability problem but as a control problem in which the system design should prevent or control unsafe behavior.

The standard method of studying adverse events in hospitals uses root cause analysis, which analyzes mistakes people make without going further. This does not provide the information needed to prevent future losses. Most people try to do the right thing in most circumstances, and mistakes are recognized frequently with the benefit of hindsight. ${ }^{6}$ The 
TABLE 1. Preoperative time-out checklist

What patient do we have?

What procedure are we doing?

What side/site/level is to be done?

Is the site marked?

Do we require any equipment, implants, radiology films, or are there any

special requirements for this patient?

Are antibiotics required?

Is DVT prophylaxis (anticoagulation) indicated?

Are there any precautions based on patient status or medications?

Suction Pre-use Checklist completed?

"If anyone has any concerns about this patient at any point during the procedure, I expect you to speak up."

$D V T$, Deep venous thrombosis.

fundamental philosophy of CAST is to understand why certain actions were carried out and the context behind that behavior.

The context of the situation greatly affects the behavior of the participants. The first step is to identify the contextual influences that determined or influenced a certain behavior. Changing the context therefore changes the behavior pattern. This systems-based approach is used in reducing accidents.

Mental models of the state of the process being controlled affect behavior. A simple feedback loop is shown in Figure 1. These figures are similar to those published earlier by our group. ${ }^{7}$ The controller may be the surgeon or nurse who executes control actions, which may be instructions or physical actions on the controlled process. The model the controller has of the current state of the controlled process affects decisions. Mistaken and perhaps unsafe behavior may result if the model of the controlled process becomes inconsistent with the real state of the process (perhaps because of missing or incorrect feedback). An example is the mistaken belief of the nurse or physician that an immunosuppressant has already been administered, when it has not been given, and, therefore, neither of them administers it.

The individual feedback control loops are part of a larger hierarchical control structure. Figure 2 shows a model of the control system (feedback and communication loops) used to control surgical medication errors at the hospital where the adverse events occurred. The model shows the system as it is assumed to work under ideal conditions. It differs for each hospital, depending on the particular processes used. Accidents and incidents occur when the control structure (ie, the designed controls) does not enforce the safety constraints on the system operation, assuming that the controller did not intentionally harm the patient.

Specific responsibilities with respect to safety are assigned to each controller in the system. Impact of how well the safety-related responsibilities are carried out are also modeled (not all the controllers

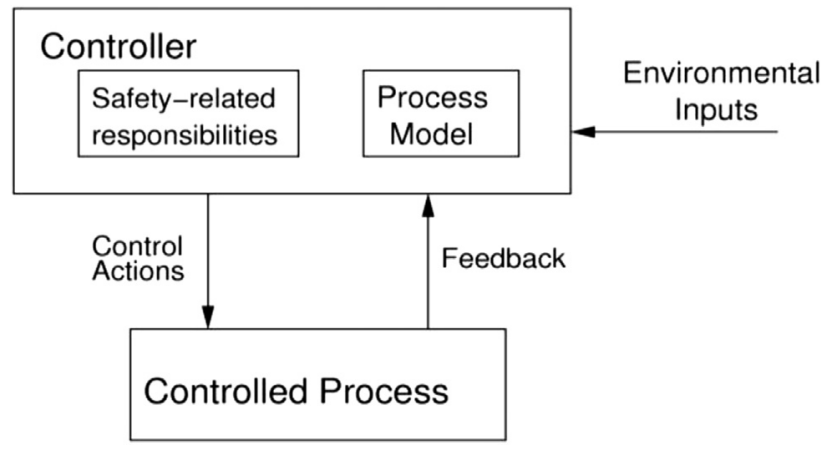

FIGURE 1. A general safety-control structure. (Reprinted with permission from Leveson et al. ${ }^{7}$ ) are shown in Figure 2). Confusion and omission of required actions could occur because the attending cardiac surgeon and the surgery fellow both have responsibility for ordering medications.

This kind of CAST analysis was conducted on each of the 30 cases identified by the Massachusetts Institute of Technology systems engineering specialists (A.L.S. and N.L.).

\section{RESULTS}

Of 380 consecutive complex cardiac surgeries, 30 adverse events occurred. Patient outcomes in those adverse events ranged from patient death to prolonged anesthetic time with no clinically observable consequences; outcomes are tabulated in Table 2. In all of these cases, $100 \%$ checklist compliance was documented by the nursing staff.

Incidents fell into several different categories, collated in Table 3. The CAST analyses on these incidents identified ways to improve the checklists. They also identified additional protection needed to prevent events that cannot be consistently prevented by using a checklist. Although the symptoms (eg, specific adverse events) differed greatly among the categories, the systemic causal factors were similar, therefore showing that fixing a few systemic factors can reduce whole categories of adverse events.

\section{Missing Medications}

There were 4 instances of missing medications: 3 cases of missing immunosuppression preoperatively and 1 case of a delay in heparin dosing. In the cases of missing immunosuppression, all of the patients had orders written for immunosuppression but somehow never received it before entering the OR. A major driver of these incidents was a lack of feedback specifically related to the electronic health record (EHR) and the time-out. The immunosuppression was ordered the night before surgery as part of a preoperative order set. This order set includes a combination of orders to be given at different times and by different people. Some orders are meant to be carried out by the intensive care nurse an hour before the patient goes to surgery, and others, such as antibiotics, are meant to be carried out by the anesthesiologist in the OR. Usually, this ambiguity is not a problem because the teams are used to carrying out these orders. These scenarios were further complicated because cardiac transplants were relatively infrequent until a recent change in leadership. The intensive care nurses had not given immunosuppression before and likely did not realize that this was their responsibility. In addition, immunosuppression was performed after hours, when the pharmacy dispensing medications was at a remote location.

Compounding the lack of feedback is the difficulty in seeing whether an order has been carried out in the EHR. To see this, one has to compare the orders with the 


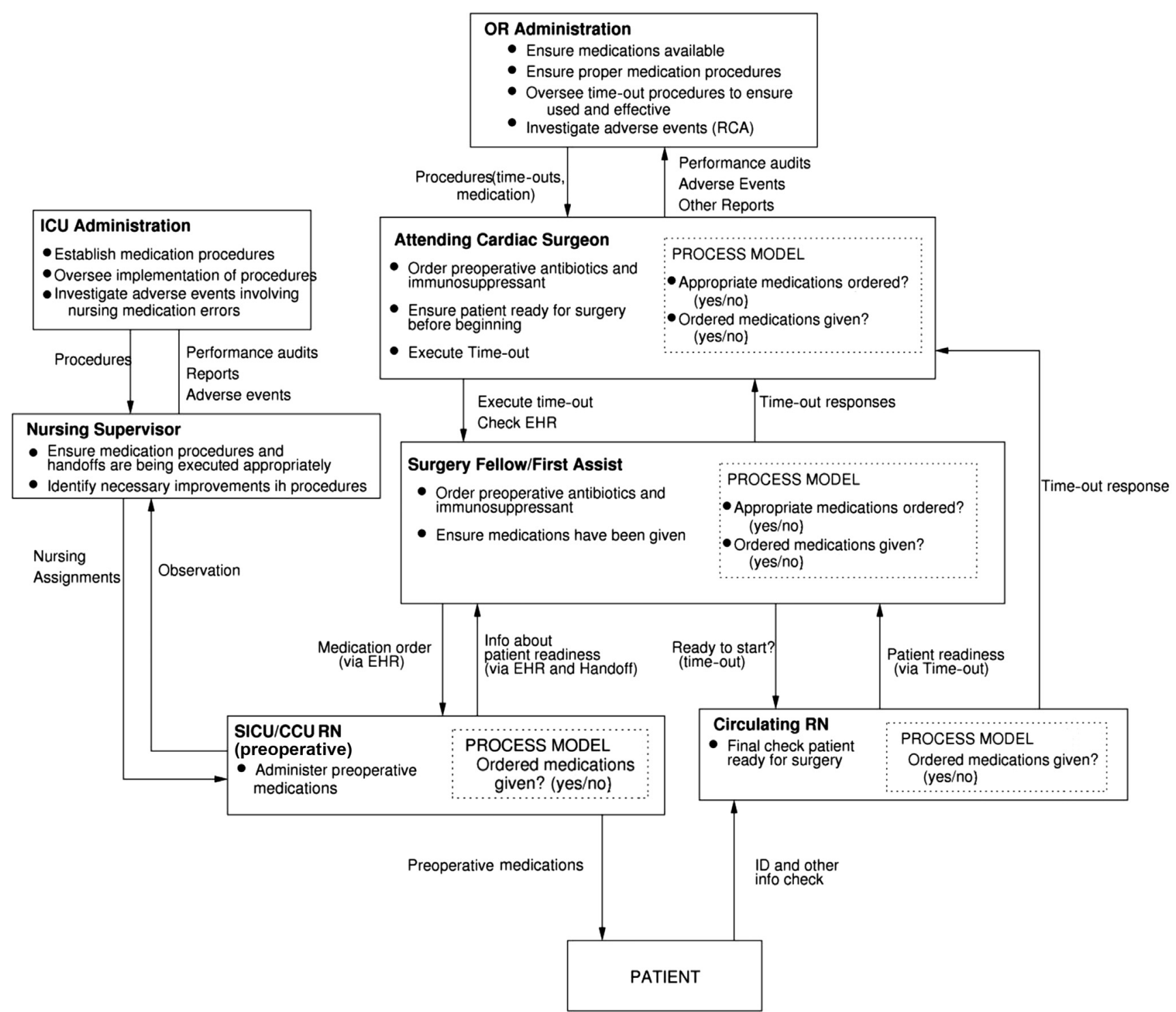

FIGURE 2. The safety-control structure to protect against preoperative medication errors. $O R$, Operating room; $R C A$, root cause analysis; $I C U$, intensive care unit; $E H R$, electronic health record; SICU, surgical intensive care unit; $C C U$, coronary care unit; $R N$, registered nurse. (Reprinted from with permission from Leveson et al. ${ }^{7}$ )

Medication Administration Record, which is on a separate screen. There was no obvious signal to the surgical team that an order had been placed but not filled. These gaps were identified a few months later, after detailed searching of the EMR by the bio-informatics expert (M.O.), focusing

TABLE 2. Adverse event outcomes and incidences

\begin{tabular}{lc}
\hline \multicolumn{1}{c}{ Patient outcomes } & Number $(\%)$ \\
\hline Death & $2(7.7)$ \\
Prolonged hospitalization & $1(3.8)$ \\
Prolonged on-pump time & $3(11.5)$ \\
Prolonged anesthetic (off-pump) & $16(61.5)$ \\
Aborted procedure & $2(7.7)$ \\
No clinical or subclinical consequences & $2(7.7)$ \\
\hline
\end{tabular}

on the times when the immunosuppressive medications were truly administered.

\section{Missing Equipment and Missing Implants}

Of the 30 incidents, 8 involved missing equipment and an additional 3 involved missing implants. Typically, missing equipment cases involved less common procedures in which the setup was missing a specialized piece of equipment. Cases involving missing implants were all valve replacement cases in which the surgical team could not obtain a properly sized valve. Cases arising from missing equipment typically shared similar proximal events with a wide variety of contributing factors. The proximal events 
TABLE 3. Adverse event categories and incidence

\begin{tabular}{lc}
\hline \multicolumn{1}{c}{ Incident category } & Number (\%) \\
\hline $\begin{array}{l}\text { Miscommunication during staff handoff throughout } \\
\text { the procedure }\end{array}$ & $4(13.3)$ \\
Missing medication before incision & $4(13.3)$ \\
Missing instrumentation leading to intraoperative delay & $8(26.7)$ \\
Missing implants leading to delays and suboptimal & $3(10.0)$ \\
$\quad$ implants being used & \\
Broken or improperly handled specialized instruments & $9(30.0)$ \\
Miscellaneous incidents & $2(13.3)$ \\
\hline
\end{tabular}

were that either the physician requested the wrong equipment or the nurse did not retrieve all of the equipment or the correct equipment for the case.

\section{Relieving Nurse Unaware of Cardiac Procedures}

There were 4 incidents in which the relieving circulating nurse did not have the skill set necessary to work in the cardiac surgery OR. The nurses did not know where to find or how to use specialized cardiac equipment.

\section{Improperly Handled Equipment}

There were 9 instances of mishandled equipment. The OR staff frequently incorrectly believed that the equipment was broken, when in reality it was set up improperly. It is easy to say that the nurse was responsible, but the biomedical engineer and the surgeon also believed the equipment to be broken. In most of these cases, it was not until after the procedure when the team met with the company representative that they realized that the device was set up incorrectly. These incidents raise the question of device design and training.

\section{Analysis of Recommendations}

In analyzing these incidents, we came up with recommendations for preventing future accidents based on the identified causal factors. An average of 3.9 recommendations were generated for each accident scenario. We further analyzed these recommendations by coding them using the VA Action Hierarchy. ${ }^{8}$ The VA Action Hierarchy is a set of guidelines to categorize preventive actions as stronger, intermediate, or weaker actions. Stronger actions include forcing functions and active leadership engagement and action, whereas weaker actions include double checks and training. Of the recommendations generated from these analyses, $35 \%$ were stronger actions, $27.5 \%$ intermediate, and $37.5 \%$ weaker.

\section{DISCUSSION}

In this research, we examined 30 adverse events that occurred during cardiac surgery on 380 consecutive patients over a 24-month period in a large American academic medical center. A time-out was conducted in a standard fashion for all patients in accordance with the WHO surgical checklist protocol. Compliance in performing the time-out was established by direct observation during the surgeries. We then used a sophisticated causal analysis method $^{6}$ to identify why the checklist did not prevent the adverse events and what else is needed to substantially reduce adverse events. However, stopping the analysis at the proximal event provides no useful information to prevent this from happening again. When we further explored why the local actors performed the wrong actions, a more nuanced picture became clear. The surgeons' pick lists, in which they list their preferred equipment for each surgery, are frequently outdated. Furthermore, many physicians are unaware that they are outdated and unaware of how to change them, suggesting that the surgical leadership team needs to enforce the updating of pick lists. In addition, there were potential problems with incomplete equipment kits, an issue that should be addressed by further investigating the entire equipment inventory and sterilization process, as opposed to blaming the nurses for not obtaining all of the required equipment.

TABLE 4. Select recommendations to supplement checklists coded by the VA Action Hierarchy

\section{Local levels}

Change the electronic health record format to provide more feedback on order status (intermediate)

Institute a formal preoperative patient handoff from surgical intensive care unit to the surgical team (intermediate)

Change the format of the pick list for surgeons' equipment preferences (intermediate)

Standardize equipment names to facilitate communication (intermediate)

Maintain a stock of blood products in the intensive care unit for emergencies (stronger)

Standardize consult procedures (stronger)

Implement novel surgical volume prediction tools to better match needed staffing levels (intermediate)
Higher control levels

Implement an incident reporting system and formal event analysis program (stronger)

Implement and enforce a policy requiring pick lists to be reviewed and updated yearly (weaker)

Institute yearly competency measures to evaluate staff training needs (weaker)

Implement weekly meetings with nursing and surgical management (and surgical and medical management) to facilitate interprofessional communication (intermediate)

Create consistent national reporting guidelines for medical device incidents (weaker)

Push medical device vendors to create more usable and safer equipment (stronger)

Make safety a top priority for the health system from the highest levels of management down (stronger) 
Analyzing these accidents with CAST provided insight not only into the limitations of checklists and how they need to be supplemented to prevent more adverse events but also, fundamentally, into the limitations of defense-indepth thinking for modeling and controlling health care risks. As 1 example, the government of Ontario, Canada, recently mandated implementation of surgical safety checklists at all hospitals in an effort to improve patient safety, as the insertion of an additional layer of defense against adverse events. In this diverse population, the WHO surgical checklist failed to reduce mortality and morbidity in a wide range of surgical patients. ${ }^{4}$ This study raises the question of how much of the early positive results were the result of the Hawthorne effect (a phenomenon that occurs when the subjects of a study alter their behavior as a response to being observed) and other systemic changes that accompanied the introduction of checklists, rather than the checklist itself.

Some of the arguments for checklists come from their use in aviation and their supposed influence on the low accident rate in that industry. However, the role and impact of checklists on aviation safety have been exaggerated. The misuse and nonuse of checklists by flight crews, aircraft maintenance workers, and operators in nuclear power plants (another industry that uses checklists) has been a major contributor to accidents or serious incidents. ${ }^{9}$ Typical pilot errors in using checklists include skipping items, often with the intention of coming back to them; interruptions and distractions; misperception (when the pilots see the checklist item in an improper status but perceive it as having the correct status); time constraints and production pressures; and incomplete compliance. ${ }^{10}$

Although checklists and standardized procedures do play a role in aviation safety, their use is a small part of the reason for the low accident rate in flying. For example, aircraft are designed using a fail-safe principle so that failures of physical components, human errors, or flaws in implementing operational procedures (including checklists) do not, by themselves, lead to an accident. The success of checklists in aviation depends on the careful analysis and design that go into the entire system design, as well as on human ingenuity in selecting and applying and even modifying standard procedures and checklists. That is, checklists are effective in commercial aviation only because the larger system is engineered to protect against human fallibility. Attributing the success of aviation safety to the use of checklists or placing too much reliance on them for health care safety would be a tremendous mistake.

The overuse of checklists without making system changes is beginning to be recognized in health care too. Stock and Sundt ${ }^{11}$ recently argued in an editorial that more than just checklists are needed to prevent accidents. In addition, there is a need to show that checklists help avoid major adverse events, otherwise surgical teams may view them just as wasteful impositions. ${ }^{12}$ Accidents are not only caused by lapses in memory, so a tool that is designed to serve as a memory aid does not protect patients throughout their hospital stay.

As it relates to missing equipment, it might be best to ask about specific equipment that is different from what is used in more routine cases. The specific question on the time-out is phrased as "Do we require any equipment, implants, radiology films, or are there any special requirements for this patient?" The question is too general to serve as a memory aid by highlighting any particular piece of missing equipment. In addition, it is a stacked question, meaning that 1 question asks for multiple answers. Studies of human factors show that stacked questions like this make it more likely that respondents miss specific parts of the general question. ${ }^{12}$

There were instances in which cross-covering nurses were not familiar with cardiac procedures. It is easy to say that the nurses were inadequately trained and that it was a problem of just a few inadequately trained personnel. However, the same incident happened 4 times with 4 different nurses and saying that it was simply a matter of poorly trained nurses does not prevent this from happening a fifth time or more. These incidents were reported to the nursing hierarchy and assurances obtained about avoiding repetitions. Adequate training and competency verification of staff in complex cardiac surgery suites falls to the institutional clinical educational department, with monthly review to keep staff properly trained.

Identifying the systemic factors involved and fixing these factors requires moving to higher levels of control in the system. Why were these nurses, who were not trained in cardiac surgery, working in the cardiac surgery ORs? Analysis of the managerial levels highlights that there is a policy against noncardiac nurses being assigned to the cardiac rooms, even as relieving nurses. However, this policy is balanced by budgetary and staffing constraints. There is a constant message from upper level management that making staff work overtime breaks the budget and hurts the organization. How, then, is the nurse manager supposed to keep the OR staffed with appropriately trained staff? These conflicting system goals (safety through using appropriately trained staff and financial constraints limiting overtime payment) need to be discussed and prioritized at the highest levels of hospital leadership before this problem is truly prevented in the future. In terms of missing equipment such as valve implants, these were related to recent use of the sized implants. 
Alternative implants made by a different manufacturer were used. Feedback was provided to the nursing hierarchy and the supply chain management group in each instance. Additional redundancy measures were introduced, such as immediate reordering of the valve implant by the circulating nurse and cross-checking of all implants before commencement of cases.

Medical devices are notoriously designed with little thought given to the usability and their integration into the existing work flow. Equipment should have as simple a design as possible, with clear labels and diagrams. Solutions that focus on making the equipment less error prone would prevent similar incidents in the future. Why, then, is medical equipment not designed using knowledge of human factors and error proneness? Medical device companies have little incentive to change the design if health systems buy the devices regardless of the design. The companies may not even know about the incidents raised by their equipment without this type of feedback. Doctors and hospitals need to start pushing for better designs and stop settling for products that they know have design flaws regardless of the time and budget pressure placed on these purchases.

The checklist and time-out are designed to be communication tools common to all ORs. Therefore, at this medical center, every OR uses the same time-out checklist, a modified WHO Surgical Safety Checklist. However, because this is a general checklist, there is no question specifically asking about immunosuppression. The way that it is currently designed, the time-out is not an effective tool for catching this type of error.

One lesson that can be learned is that checklists need to be tailored to the specific task being performed, in this case cardiac surgery. Questions about deep vein thrombosis prophylaxis may have limited relevance, for example. The rote use of stock questions may take up time and decrease the likelihood that key OR personnel are fully attentive to the entire checklist. Additional questions that are relevant (eg, about preoperative immunosuppression in some of these cases) need to be added. However, adding questions is a difficult task. There is a compromise between adding questions to cover more content and making the checklist so long that the user does not complete it. There need to be changes to the format of the checklist to increase its usability. These changes include separating stacked questions and creating more close-ended questions.

However, making these changes to the checklist would not have prevented all the adverse events. The CAST analysis discovered systemic causes at both the local level as well as at higher levels of the system safety-control structure. For example, management of change procedures need to be instituted and used when changes are made in standard practices, such as, in this case, an increase in cardiac transplant surgery. Risks of changes should be evaluated and proper design of procedures and instruction provided to ensure that new risks are not introduced by the change.

Other recommended changes are listed in Table 4. Many of the higher control level recommendations would prevent accidents of many different types. For example, implementing a strong incident reporting system with formal investigations may have prevented the repetitive incidents. The UO (unexpected occurrence) forms were infrequently filled out in these 30 adverse events, suggesting that the mechanisms set in place by the administration were not being used properly. Would unsafe staffing levels be a problem if safety took priority over cost in all managerial decision making? In addition, many of the identified changes with these analyses fell into the stronger actions categorization, suggesting that they would be more effective at preventing these accidents in the future than merely training or reminding staff of how to properly perform their jobs. Until these changes at all levels of the system are made to eliminate the systemic factors in adverse events, we will continue to see problems at the lower levels with unsafe staffing, inadequate training, insufficient stock, poor design of equipment and computer records, and blood banks located a block away from where blood is most needed.

We have good evidence to show that process improvement measures initiated by team members, even at the level of a fellow or trainee, may have great implications in improving perioperative outcomes. ${ }^{13}$ Focusing on reducing preventable adverse events may by itself not be as important as working through the processes to improve perioperative outcomes. ${ }^{14}$ When the adverse events do occur, adequate mechanisms should be in place to rescue these patients from complications, because measuring failure to rescue may be a better metric than looking at adverse events alone. ${ }^{15}$

From our experience with this CAST approach, we made some changes in our practice:

1. Customized time-out and checklist for complex cardiac surgery with separate set of questions for transplants and VADs

2. Cross-checking the administration/delivery of preoperative medications in all cardiac surgery procedures

3. A preoperative check with the nurses about possible range of implants being used for the case

4. Ensuring availability of specialized equipment, before the case.

It is our plan to repeat this study to evaluate effects of these changes, over a 2-year period. 


\section{CONCLUSIONS}

Time-outs and checklists can play a role in patient safety. However, Their use in these cases did not prevent the adverse events that occurred. The fact that accidents happen despite having implemented a preoperative checklist goes beyond merely an issue with checklist compliance or even checklist design. Part of the solution is to improve the checklists, as suggested in this study and others. However, an improved checklist does not prevent most of the causal factors identified in this research and may not be the best way to solve them even if it could. Solutions need to move beyond the local level at which the checklist acts and into the overall system design and controls to truly be effective. These changes would have the added benefit of improving care throughout the entire health system and not just surgical care.

A checklist is only one of the tools in our arsenal for improving patient safety. Identifying the systemic factors in adverse events and correcting them could have a major impact on patient safety.

\section{Conflict of Interest Statement}

We gratefully acknowledge the support of the Portuguese Science and Technology Foundation (FCT) through the MIT Portugal Program. MIT Portugal had no role in the design and conduct of the study; collection, management, analysis, and interpretation of the data, or preparation, review, or approval of the manuscript. Authors have nothing to disclose with regard to commercial support.

J.R. and N.L. had full access to all the data in the study and take responsibility for the integrity of the data and the accuracy of the data analysis.

\section{References}

1. Haynes AB, Weiser TG, Berry WR, Lipsitz SR, Breizat AS, Delling EP, et al. A surgical safety checklist to reduce morbidity and mortality in a global population. N Engl J Med. 2009;360:491-9.

2. de Vries EN, Prins HA, Rogier C, den Outer AJ, van Andal G, van Helden SH, et al. Effect of a comprehensive surgical safety system on patient outcomes. $N$ Engl J Med. 2010;363:1928-37.

3. Ko HC, Turner TJ, Finnigan MA. Systematic review of safety checklists for use by medical care teams in acute hospital settings-limited evidence of effectiveness. BMC Health Serv Res. 2011;11:211.

4. Urbach DR, Govindarajan A, Saskin R, Wilton AS, Baxter NN. Introduction of surgical safety checklists in Ontario, Canada. N Engl J Med. 2014;370: 1029-38.

5. Rydenfält C, Johansson G, Odenrick P, Akerman K, Larsson PA. Compliance with the WHO Surgical Safety Checklist: deviations and possible improvements. Int J Qual Health Care. 2013;25:182-7.

6. Dekker S. Discontinuity and disaster: gaps and the negotiation of culpability in medication delivery. J Law Med Ethics. 2007;35:463-70.

7. Leveson N, Samost A, Dekker S, Finkelstein S, Raman J. A systems approach to analyzing and preventing hospital adverse events. J Patient Saf. January 11, 2016 [E-pub ahead of print].

8. VA National Center for Patient Safety. Root Cause Analysis Tools. 2015:30. Available at: http://www.patientsafety.va.gov/professionals/onthejob/rca.asp. Accessed August 26, 2015.

9. Leveson NG. Engineering a Safer World. Cambridge, MA: MIT Press; 2011: 534.

10. Degani A, Wiener EL. Cockpit checklists: concepts, design, and use. Hum Factors. 1993:35:28-43.

11. Stock CT, Sundt T. Timeout for checklists? Ann Surg. 2015;261:841-2.

12. Catchpole KR, Dale TJ, Hirst DG, Smith JP, Giddings TA. A multicenter trial of aviation-style training for surgical teams. J Patient Saf. 2010;6:180-6.

13. Loor G, Vivacqua A, Sabik JF III, Li L, Hixson ED, Blackstone EH, Koch CG. Process improvement in cardiac surgery: development and implementation of a reoperation for bleeding checklist. J Thorac Cardiovasc Surg. 2013;146: 1028-32.

14. Shojania KG, Mheen PJM. Temporal trends in patient safety in the Netherlands: reduction in preventable adverse events of the end of adverse events as useful metric? BMY Qual Saf. 2015;24:541-4.

15. Ahmed EO, Butler R, Novick RJ. Failure-to-rescue rate as a measure of quality of care in a cardiac surgery recovery unit: a five-year study. Ann Thorac Surg. 2014; 97:147-52.

Key Words: checklists, time-out, perioperative, adverse events, systems analysis 